\title{
Good recovery of a patient with neurocysticercosis using two antihelminthic drugs combined with steroid
}

\author{
Xin-Di Li, Hua-Bing Wang, Heng Zhou, An-Na Zhou, Lin Zhao, Yong-Hong Liu, Xiao-Qing Gong, Xing-Hu Zhang
}

Department of Neurology, Beijing Tiantan Hospital, Capital Medical University, Beijing 100050, China.

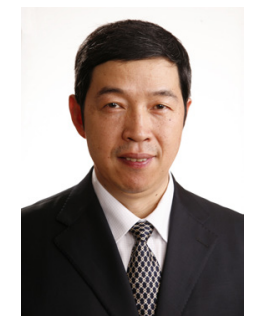

\begin{abstract}
Mr. Xing-Hu Zhang, MD, Professor, is Director of Neuroinfection and Neuroimmunology Center, Department of Neurology, Beijing Tiantan Hospital, Capital Medical University, China. He is a member of Neuroimmunology Group, Neurology Branch, Chinese Medical Association, and a member of Neuroimmunology Branch, Chinese Society of Immunology. And also, he is a member of Editorial Board of Journal of Chinese Neuroimmunology \& Neurology.
\end{abstract}

\begin{abstract}
Neurocysticercosis is the most common parasitic infection of the central nervous system. We present a case report of a neurocysticercosis patient with multiple cysts, who presented with new onset generalized tonic-clonic seizures. A 4-cycle treatment of 2 different antihelminthic drugs with dexamethasone and sodium valproate led to clinical improvement without any adverse reactions. The manifestations of neurocysticercosis are protean and the diagnosis should be considered whenever multiple cysts are seen on computed tomography or magnetic resonance imaging. The antihelminthic treatment of neurocysticercosis should be individualized, especially for patients with multiple cysts.
\end{abstract}

Key words: Neurocysticercosis; parenchymal neurocysticercosis; neurocysticercosis diagnosis; antihelminthic drug

\section{INTRODUCTION}

Neurocysticercosis has become a serious public health concern, especially in developing countries where the prevalence rate reaches $4 \% \cdot{ }^{[1]}$ Neurocysticercosisis caused by the larval form of the tapeworm Taenia solium grow in cerebral parenchyma, ventricles and subarachnoid space, and is the leading cause of acquired epilepsy. Therapeutic measures include surgery, symptomatic therapy and antihelminthic drugs such as albendazole and praziquantel. ${ }^{[2]}$ Evidence supporting existing treatment guidelines for neurocysticercosis is inadequate although there has been a comparison between the efficacy of albendazole and praziquantel. ${ }^{[2]}$

Corresponding Author: Dr. Xing-Hu Zhang, Department of Neurology, Beijing Tiantan Hospital, Capital Medical University, No.6 Tiantanxili, Dongcheng District, Beijing 100050, China. E-mail:xhzhtiantan@hotmail.com

\begin{tabular}{|l|l|}
\hline \multicolumn{2}{|c|}{ Access this article online } \\
\hline Quick Response Code: & Website: \\
\hline & www.nnjournal.net \\
\cline { 2 - 2 } & \\
& DOI: $10.20517 / 2347-8659.2015 .51$ \\
\hline
\end{tabular}

\section{CASE REPORT}

A 45-year-old male was brought to a local hospital with a generalized tonic-clonic seizure which self-terminated after $2 \mathrm{~min}$. Further such seizures recurred over the following 20 days. There was no headache, dizziness or vomiting. His weight was $80 \mathrm{~kg}$. On examination of the central nervous system, higher mental functions were normal (30/30 mini-mental state examination). All cranial nerves were intact. All muscles tone and power, deep tendon and planter reflexes were all normal. The general examination was normal. The first brain magnetic resonance imaging (MRI) [Figure 1], undertaken at the local hospital, showed multiple large spherical viable cysts distributed evenly throughout the brain parenchyma, that were hyperintense (with respect to brain parenchyma) on the T2-weighted scan and

This is an open access article distributed under the terms of the Creative Commons Attribution-NonCommercial-ShareAlike 3.0 License, which allows others to remix, tweak, and build upon the worknon-commercially, as long as the author is credited and the new creations are licensed under the identical terms.

For reprints contact: service@oaepublish.com

Cite this article as: Li XD, Wang HB, Zhou H, Zhou AN, Zhao L, Liu YH, Gong XQ, Zhang XH. Good recovery of a patient with neurocysticercosis using two antihelminthic drugs combined with steroid. Neuroimmunol Neuroinflammation 2016;3:120-3.

Received: 17-11-2015; Accepted: 06-03-2016 

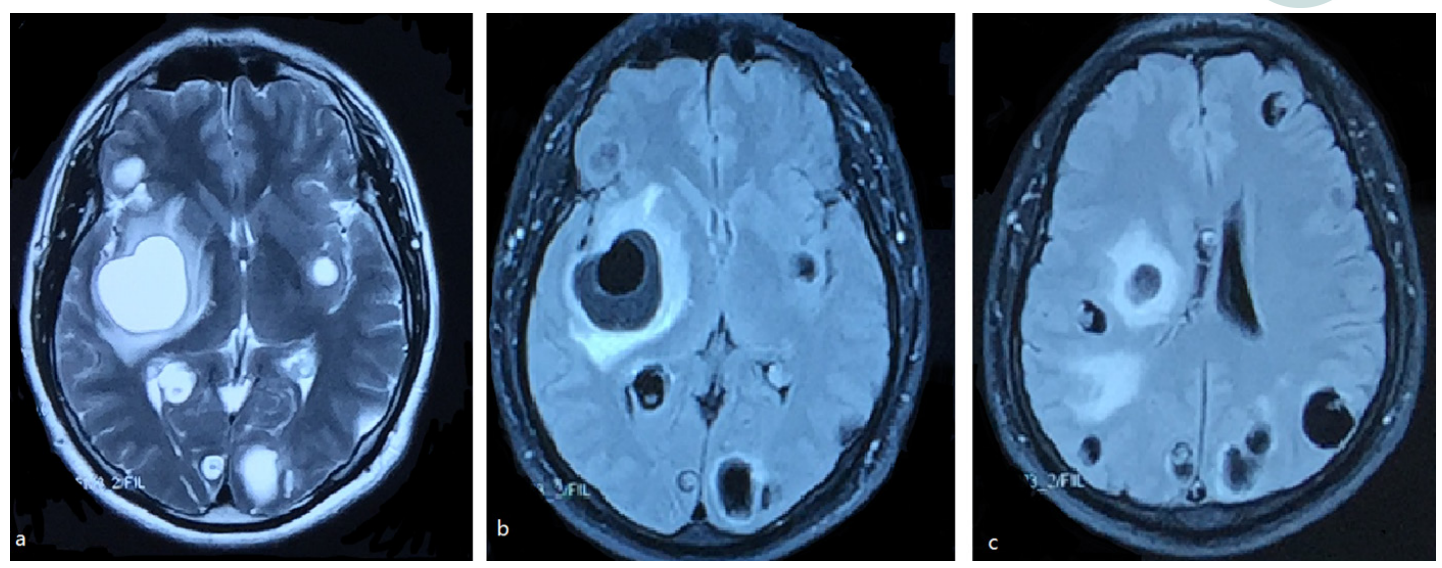

Figure 1: Brain magnetic resonance imaging from the local hospital showed multiple large cysts accompanied by perilesional oedema, in the presence of scolex (a: T2-weighted image; b and c: Flair image)

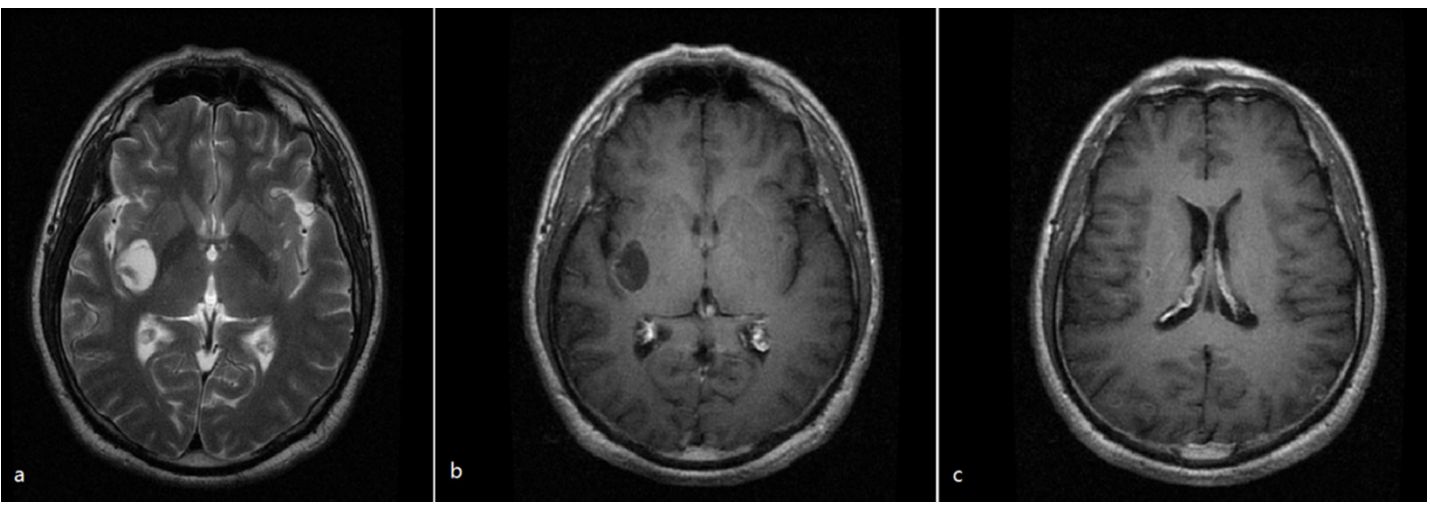

Figure 2: Magnetic resonance imaging (MRI) after 2 cycles of treatment with albendazole. (a) T2-MRI and gadolinium-enhanced MRI; (b) showed a single large cyst with cerebrospinal fluid-like signal in the right basal ganglia region; (c) gadolinium-enhanced MRI showed some ring-like or nodular contrast enhancements in occipital lobe
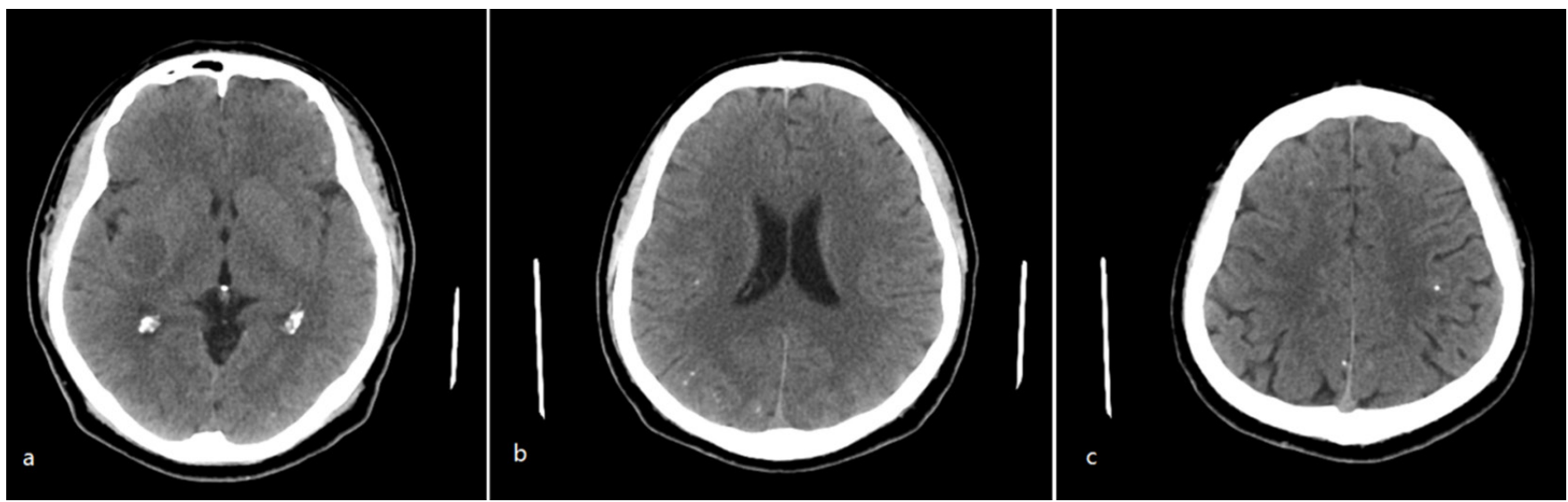

Figure 3: Computed tomography after 2 cycles of treatment with albendazole. There was a single large hypointense cyst in the right basal ganglion region (a) and the resolution of other cysts associated with calcification around the lateral ventricles (b) and in the cerebral cortex(c)

hypointense on the T1-weighted scan with ring enhancement. The patient was transferred to the Beijing Tiantan Hospital for diagnosis and treatment. Examination findings remained unremarkable. Blood cell counts and erythrocyte sedimentation rate were within the normal range. Cerebrospinal fluid analysis revealed an antibody of Taenia solium. On this basis, the patient was diagnosed with neurocysticercosis. Dexamethasone $20 \mathrm{mg} /$ day was initially used and reduced to $10 \mathrm{mg} /$ day after 6 days. Later, sodium valproate $(500 \mathrm{mg}$ bid) was added. Albendazole was given at a dose of 400$1,200 \mathrm{mg} /$ day as the antihelminthic treatment for 17 days. After 1 month of treatment, the generalized seizures remitted and the patient's condition improved. He was discharged on day 23.

At follow-up 6 months later, the patient started to use albendazole again at a dose of $400 \mathrm{mg} /$ day for 2 days, then added to $800 \mathrm{mg} /$ day for 4 days, finally increased to $1,200 \mathrm{mg} /$ day for 10 days. At 2 months, clinical recovery was accompanied by neuroimaging improvement. Most cysts have reduced in diameter, and 1 large cyst persisted in the right basal ganglia. These lesions showed ring-like or nodular contrast enhancement on brain MRI [Figure 2] and calcification on the brain computed tomography [Figure 3]. 

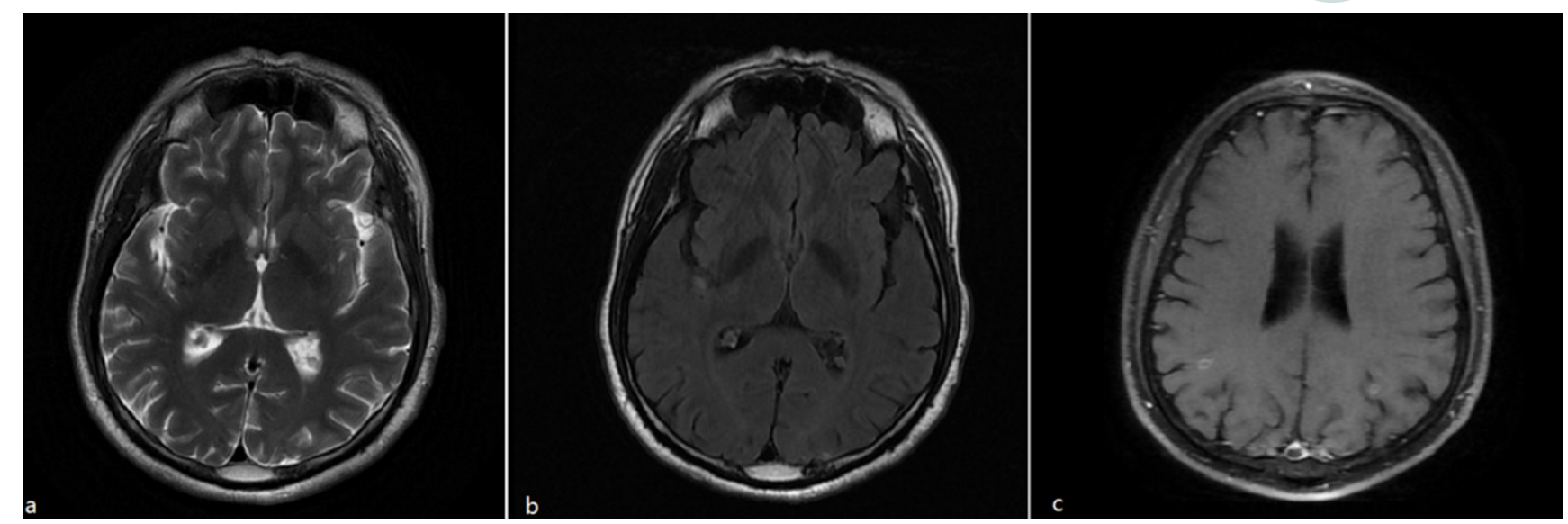

Figure 4: Magnetic resonance imaging at the end of treatment. All cysts were resolved on T2-weighted image (a) and Flair image(b). But a few ring-like or nodular contrast enhancements in the occipital lobe were found on gadolinium-enhanced MRI

Sixteen months after the original presentation, the patient was re-admitted to Beijing Tiantan hospital after a generalized tonic-clonic seizure and reporting formication with parasthesia on the left limbs for 2 months. Instead of albendazole, he was given praziquantel at an initial dose of $400 \mathrm{mg} /$ day increasing to $1,800 \mathrm{mg} /$ day $(400 \mathrm{mg} /$ day for 1 day, $600 \mathrm{mg} /$ day for 4 days, $1,200 \mathrm{mg} /$ day for 9 days and 1,800 mg/day for 2 days). He responded with no further seizures. 2 months after the therapy, the brain MRI revealed that the large right basal ganglia cyst had reduced in size.

He went back to our hospital after 4 months for further evaluation of his illness. We suggested another cycle praziquantel ( $600 \mathrm{mg} /$ day for 3 days and 1,800 mg/day for 10 days). Then he was in a stable status and discharged. A brain MRI [Figure 4] showed complete resolution of the large cyst. He continued on the sodium valproate.

\section{DISCUSSION}

The clinical manifestations of neurocysticercosis are poorly specific without a "typical" syndrome owing to the variable factors including the number, type, size, localization of cysticerci, as well as the host immune response against the parasite. Our patient presented with a generalized tonic-clonic seizure, which in case series is the most frequent clinical manifestation of intraparenchymal neurocysticercosis. ${ }^{[3]}$ Seizures secondary to neurocysticercosis are attributed to the local cortical inflammation arising from the death of the cysticercus, or gliosis associated with end-stage calcified lesions. A literature review states that $50 \%$ of patients with neurocysticercosis develop recurrent seizures. ${ }^{[4]}$ According to the major diagnostic criteria, neurocysticercosisis diagnosed in the presence of suggestive lesions on neuroimaging studies (images of cyst and scolex) and positive serum anticysticercal antibodies detected by enzyme-linked immuno sorbent assay ${ }^{[5]}$ Our patient's earliest brain MRI was typical for neurocysticercosis, but it was unrecognised by the local hospital. Delayed diagnosis contributes to extensive greater burden of neurological and psychiatric morbidity, and so awareness of neurocysticercosis should be raised especially in regional primary hospitals. The detection of antibodies to Taenia solium glycoprotein antigens can support the diagnosis but, where negative, does not exclude neurocysticercosis.

Management of neurocysticercosis includes surgery, symptomatic therapy and antihelminthic drugs. The patient's outcome is evaluated according to the improvement of symptoms and lesion alleviation on neuroimaging studies such as the disappearance of perilesional oedema and reduction of number of viable cysts. There is no standard definition of what constitutes a resolved cyst. In accordance with a recent randomised controlled trial, ${ }^{[6]}$ it is accepted that the absence of discernible hyperintense contents on T2 MRI could be the final marker of parasite degeneration. In the case presented, the MRI scans were undertaken approximately 2 months after the end of each cycle of treatment to evaluate the effect of the treatment. Surgery is thought primarily as an option for treating intraventricular cysts and so was not indicated in this case. Symptomatic therapy included antiepileptic drugs and corticosteroids. For our patient, we gave dexamethasone in order to attenuate the parenchymal inflammatory reaction, and sodium valproate to control the clinical seizures. Then we added an antihelminthic drug. Albendazole is a broad-spectrum antihelminthic drug. According to a recent meta-analysis of randomized trial, albendazole use is associated with resolution of active cysts and fewer generalized seizures in patients with parenchymal neurocysticercosis. ${ }^{[7]}$ It is also reported that albendazole is particularly indicated for symptomatic patients presenting with multiple viable brain parenchymal cysticerci. Compared to patients with extraparenchymal lesions, albendazole is more effective in patients with parenchymal lesions..$^{[8,9]}$ The 
usual recommended dose for albendazole is $15 \mathrm{mg} / \mathrm{kg}$ per day divided into two doses every $12 \mathrm{~h}$ for 1 week while the duration of antihelminthic therapy varies. ${ }^{[10]}$ In this case, our patient started on albendazole at a low dose when he had multiple parenchymal cysts. After a 2-cycle treatment of albendazole, the perilesional oedema faded away, the number of large cysts reduced to one, and most of the cysts became calcified nodules. In contrast with most neurocysticercosis cases, where only one type of antihelminthic drugs is given, we utilized praziquantel to target a single unremitting large cyst. Praziquantel can destroy cysticercus cysts in parenchymal brain by changing the metabolism of intracellular calcium. It has been pointed out that praziquantel is favoured in patients with lower cyst burdens, while the multiple cysts barely changed after short course of praziquantel introduced by Pretell et al. ${ }^{[11]}$ In contrast to albendazole, praziquantel is associated with more frequent adverse reactions (e.g. headache and vomiting). However, after another 2-cycle treatment of praziquantel in this case, the large cyst in the right basal ganglia region had dramatically resolved without adverse drug reactions. Recent studies about the relative efficacy of these two antihelminthic drugs seemed to demonstrate that albendazole might be the drug treatment of first choice in neurocysticercosis. While praziquantel has been shown to eliminate 60$70 \%$ of parenchymal brain cysts, albendazole has been found to destroy $80-90 \%$ of them. A meta-analysis revealed that albendazole was more effective than praziquantel regarding the clinical outcomes of patients with neurocysticercosis. ${ }^{\left[{ }^{[8]}\right.}$ A randomised controlled trial also showed the increased antihelminthic efficacy of an albendazole plus praziquantel regimen, and that further seizures were less frequent in individuals with complete cyst resolution after antihelminthic treatment. ${ }^{[6]}$ On the basis of those studies and this case report, we believe that the antihelminthic therapy should be rationally and individually based on different circumstances and that albendazole and praziquantel have equivalent efficacy at different stages of the disease. We suggest that the combination antihelminthic treatment of albendazole and praziquantel has potential benefits for patients with multiple cysticercus cysts in brain.

\section{Financial support and sponsorship}

Nil.

\section{Conflicts of interest}

There are no conflicts of interest.

\section{REFERENCES}

1. Mamkin I, Sood N, Ramanan SV. Taenia solium neurocysticercosis. NEngl JMed 2007;357:1666-7.

2. Osvaldo MT, Newton SO, Pierina SB, Jose EL, Vera LL. Medical management of neurocycsticercosis. Expert Opin Pharmacother 2011;12:2845-56.

3. Singh G1, Burneo JG, Sander JW. From seizures to epilepsy and its substrates: neurocysticercosis. Epilepsia 2013;54:783-92.

4. Nash TE, Del Brutto OH, Butman JA, Corona T, Delgado-Escueta A, Duron RM, Evans CA, Gilman RH, Gonzalez AE, Loeb JA, Medina MT, Pietsch-Escueta S, Pretell EJ, Takayanagui OM, Theodore W, Tsang VC, Garcia HH. Calcific neurocysticercosis and epileptogenesis. Neurology 2004;62:1943-8.

5. Del Brutto OH. Diagnostic criteria for neurocysticercosis,revisited. Pathog Glob Health 2012;106:299-304.

6. Garcia HH, Gonzales I, Lescano AG, Bustos JA, Zimic M, Escalante D, Saavedra H, Gavidia M, Rodriguez L, Najar E, Umeres H, Pretell EJ; Cysticercosis Working Group in Peru. Efficacy of combined antiparasitic therapy with praziquantel and albendazole for neurocysticercosis: a doubleblind, randomised controlled trial. Lancet Infect Dis 2014;14:687-95.

7. Kalra V, Dua T, Kumar V. Efficacy of albendazole and short-course dexamethasone treatment in children with 1 or 2 ring-enhancing lesions of neurocysticercosis: a randomized controlled trial. JPediar 2003;143:111-4.

8. Matthaiou DK, Panos G, Adamidi ES, Falagas ME. Albendazole versus praziquantel in the treatment of neurocysticercosis: a meta-analysis of comparative trials. PLoS Negl Trop Dis 2008;2:e194.

9. Gardenas G, Carrillo MR, Jung H, Sciutto E, Hernandez JL, Fleury A. Subarachnoidal neurocysticercosis non-reponsive to cysticidal drugs: a case series. BMC Neurol 2010;10:16.

10. Takayanagui OM. Therapy forneurocysticercosis. Expert Rev Neurother 2004:4:129-39.

11. Pretell EJ, Garcia HH, Gilman RH, Saavedra H, Martinez M; Cysticercosis Working Group in Peru. Failure of one-day praziquantel treatment in patients with multiple neurocysticerosis lesions. Clin Neurol Neurosurg 2001;103:175-7. 